\title{
Caracterización morfológica del cerdo criollo Pillareño del cantón Guamote de Ecuador
}

\author{
Olmedo, W.J. ${ }^{1}$, Toalombo, P.A. ${ }^{\circledR}{ }^{\circledR}$ Flores, L.G. ${ }^{2}$; Delgado, J.V. ${ }^{3}$; Navas, F.J. ${ }^{3,4}$; Duchi, N.A. ${ }^{2}$
}

'Investigador independiente.

${ }^{2}$ Escuela Superior de Chimborazo, Carrera de Zootecnia, Riobamba. Ecuador.

${ }^{3}$ Departamento de Genética. Universidad de Córdoba. España.

${ }^{4}$ Instituto Andaluz de Investigación y Formación Agraria, Pesquera, Alimentaria y de la Producción Ecológica. Universidad de Córdoba. España.

\section{PalABRAS CLAVE}

Acervo genético.

Medidas zoométricas.

Recursos Zoogenéticos.

Variables fanerópticas.
Índices zoométricos.

\section{RESUMEN}

Se caracterizó morfológicamente al cerdo criollo del cantón Guamote situado a 44 Km de la Ciudad de Riobamba, Provincia de Chimborazo. La investigación tuvo una duración de 60 días, se utilizó una muestra poblacional de 72 animales determinados mediante una ecuación de muestra finita; se caracterizaron 14 medidas zoométricas, 7 variables fanerópticas y 7 índices zoométricos de machos y hembras mayores a 6 meses de edad. Mediante estadística descriptiva se obtuvo medidas zoométricas con promedios de; LK 24,64 cm; LC 15, $11 \mathrm{~cm} ; A K$ 14,77cm; AO 15,71 cm; LO 19,56 cm; AC 59,54 $\mathrm{cm} ; A$ AG 65,92 cm; DL 81,61; DDE 31,40 cm; DB 26,94 cm;ANG 25,02 cm; LG 25,85 cm; PT 91,24 cm; PC 16,91 cm; y PV 59,61 kg. De acuerdo a las variables fanerópticas e índices zoométricos se concluye que el cerdo criollo perteneciente al cantón Guamote es un animal con características similares al cerdo lbérico de la variedad Gabana, con una capa totalmente negra sin manchas, mucosas oscuras, pelo largo y abundante, de perfil subcóncavo, dolicocéfalo en el cual predomina el largo de su cara sobre el ancho, de buena profundidad de pecho la cual determina su funcionalidad y rusticidad, con una grupa de líneas convexas muy bueno para la reproducción, eumétricos o de tamaño mediano con ausencia de mamellas y sindactilia, por lo cual se recomienda garantizar la conservación de cerdos criollos en el cantón Guamote, para evitar la pérdida de un acervo genético único de la zona, ya que constituye un recurso zoogenético de gran importancia para la diversidad agroecológica del Ecuador.

\section{Morphological characterization of the Creole pigs that are called Pillareño of the Canton Guamote from Ecuador}

\section{SUMMARY}

\section{ADDITIONAL KEYWORDS}

Gene pool.

Genetic resources.

Zoometric Indices.

Morphological traits.

Phaneroptic traits.

\section{INFORMATION}

\section{Cronología del artículo.}

Recibido/Received: 08.09.2020

Aceptado/Accepted: 23.03 .2021

On-line: 15.04 .2021

Correspondencia a los autores/Contact e-mail:

ptoalombo@espoch.edu.ec
We morphologically characterized the creole pig of the Guamote canton located $44 \mathrm{~km}$ from the city Riobamba, belonging to the province of Chimborazo. The investigation lasted for 60 days, during which a descriptive methodology for the morphological characterization was performed in a population sample of 72 animals, measuring a 14 zoometric and seven phaneroptical variables. Additionally, seven proportional zoom indexes of male and female pigs over six months of age. Avcerages for the zoometric measurements of the Creole pigs of the Guamote canton were; LK $24.64 \mathrm{~cm}$;C $15.11 \mathrm{~cm}$; AK 14.77cm; AO 15.71 $\mathrm{cm} ; \mathrm{LO} 19.56 \mathrm{~cm}$; AC $59.54 \mathrm{~cm}$; AG $65.92 \mathrm{~cm} ; \mathrm{DL}$ 81.61; SDD $31.40 \mathrm{~cm} ; \mathrm{DB} 26.94 \mathrm{~cm}$; ANG 25.02 $\mathrm{cm} ;$ LG $25.85 \mathrm{~cm} ;$ PT $91.24 \mathrm{~cm}$; PC $16.91 \mathrm{~cm}$; and PV $59.61 \mathrm{~kg}$. According to the panoptical variables and zoometric indexes, it is concluded that the Creole pig belonging to the Guamote canton is an animal with characteristics similar to the lberian pig of the Gabana variety with a completely black coat without spots, dark mucous membranes, long and abundant hair, in profile sub-concave, dolichocephalic in which the length of its face predominates over the width, good depth of chest which determines its functionality and rusticity, with a rump of curved lines very good for reproduction, eumétrics or medium-sized with no lamellas and syndactyly, which is why it is recommended to guarantee the conservation of Creole pigs in the Guamote canton, since it constitutes a zoogenetic resource of great importance through the generation of genetic nuclei in the area, thus allowing new generations to inherit the great wealth and diversity of the genetic resources existing in Ecuador.

\section{INTRODUCCIÓN}

El genotipo del cerdo criollo de Ecuador y de América Latina, presentan un predominio de alelos de poblaciones porcinas españolas que aportaron en su formación (Vargas, Velázquez, \& Chacón, 2015), es decir que tienen su origen en las razas ibéricas y Celtas (Ramirez et al., 2009), (Ca), que llegaron durante el período de la conquista (Benítez, 1995) y en las exploraciones realizadas en Perú y México (Rodero, Delgado, \& Rodero, 1992), por lo que se encuentran vinculados históricamente entre sí; las diferencias morfológicas son consecuencia de más de 500 años de adaptación a los diferentes sistemas agroecológicos de cada uno de los países; y que a través de los años se establecieron como grupos genéticos denominados como razas y bio- 
tipos criollos; mientras que por la FAO fueron denominados como recursos zoogenéticos (Hernández-Baca, Gámez-Rivas, \& Zeledón-Ortega, 2017).

La introducción de genotipos comerciales, han significado un importante flujo al material genético criollo (Pineda, 2006), por lo que en la actualidad se considera como un recurso zoogenético que se encuentra amenazado, a medida que la explotación de razas especializadas se incrementa y existe un mayor mestizaje por la presión que enfrenta la producción ganadera, en generar mayor cantidad de alimentos para la población a nivel mundial (Thornton, van de Steeg, Notenbaert, \& Herrero, 2009). El manejo tradicional, la adaptación a diferentes ambientes y pisos climáticos son características que no se encuentran en razas especializadas, lo cual genera una parcial eliminación del sistema primitivo de crianza y explotación animal; por tanto, este acervo genético, representa sin duda para los pueblos un valor intangible cultural y gastronómico innegable; pero a su vez económico ya que según (Zenteno, Cueva, \& Crespo, 2019) los rendimientos a la canal en cerdos autóctonos españoles estuvieron entre el 75 y 89\%, con una variabilidad muy marcada debido a la forma de faenado, edad, peso, sexo y sistema de explotación, siendo rentable para el productor. Es entonces de suma importancia conservar biotipos naturalizados, nativos y autóctonos por su alto potencial de adaptación a condiciones agroclimáticas adversas y cuyo uso está limitado comúnmente al auto consumo o ahorro familiar (Hernández-Baca et al., 2017), lo que constituye una opción en la ganadería para enfrentar la demanda de alimentos debido al crecimiento poblacional (Gutiérrez, Luna, \& Nájera, 2018).

La caracterización del cerdo criollo constituye el primer paso hacia su conservación y protección, (Estupiñán, Vasco, Barreto, \& Zambrano, 2009), para la recuperación de la genética nativa que durante décadas ha sido el sustento del pequeño productor y campesino, además que se promueve la utilización de la biodiversidad (Véliz, Mora, Barreto, \& Zambrano, 2009)contribuyendo a mejorar la seguridad, soberanía e inocuidad alimentaria, (Revidatti, 2009).

En el cantón Guamote de la provincia de Chimborazo, no existen estudios de esta índole por lo cual la información generada a partir de esta investigación permitirá identificar genotipos con amplia base genética, para incrementar su productividad (Gutiérrez et al., 2018), cuyo material genético se pueda emplear en el planes de mejoramiento; como estrategia para evitar su erosión genética.

\section{MATERIAL Y MÉTODOS}

La presente investigación se realizó en el Cantón Guamote, Provincia de Chimborazo, Ecuador; ubicado a una Altitud de $3094 \mathrm{msnm}$, temperatura $11.4^{\circ} \mathrm{C}$, Humedad relativa $72 \%$, precipitación $699 \mathrm{~mm}$. El trabajo de campo tuvo una duración de 60 días. Los animales utilizados para el ensayo fueron de ambos sexos con edades de 6 meses en adelante. Para el tamaño de la población se consideró los datos del Instituto Nacional de Estadísticas y Censos (INEC) del año 2016, que indica que se cuenta con 9758 cerdos criollos en el cantón de Guamote; el tamaño de la muestra se determinó según el criterio de (Benítez, 1995), mediante la fórmula de muestra finita, que se detalla a continuación; reduciendo el sesgo de error al 5\%, del cual se obtuvo como resultado 72 cerdos criollos.

Se midieron 15 variables zoométricas (expresadas en $\mathrm{cm}$, excepto el peso vivo que se expresa en $\mathrm{kg}$ ) y se calcularon 7 ínidces zoométricos, de acuerdo con (Aparicio, 1960) y (Sotillo \& Serrano, 1985) (Díaz, 1965), (C.M. Vietes, 1986) y recomendado por (Hurtado, 2004), (Hurtado, 2006). La metodología se detalla en la Tabla I y Tabla II.

Fórmula de muestra finita:

\section{Donde:}

\section{$\mathrm{n}=$ Tamaño de la muestra}

$\mathbf{N}=$ Total de la población

$\mathbf{Z}=$ Nivel de confianza $95 \%=1.96$

p=Proporción esperada 5\%=0,05

$\mathrm{q}=1-\mathrm{p}=0,95$

d= Precisión $5 \%=0,05$

Se recogieron datos de 6 variables cualitativas (fanerópticas) como, color de la capa (CC); color de la mucosa (KM); cobertura de pelo en el cuerpo (CP); presencia de mamelas (MM); tipo de perfil (TP); sindáctilia (CM).

Los datos se tabularon mediante estadística descriptiva, por lo que se obtuvo medidas de tendencia central: media, moda, mediana, y medidas de dispersión: desviación estándar; utilizando el programa estadístico Excel (versión 15, 2013).

Los animales que fueron parte del estudio, debían cumplir específicamente con las características descritas por (Panés, 1916), cerdo "criollo puro". Para lo cual se consideraron características de inclusión, que correspondían a las de un animal poco armónico, basto y destartalado; de peso y alzada medios; cabeza de medianas dimensiones y perfil ligeramente curvilíneo. Con amplias orejas caídas y dirigidas hacia delante; cuello relativamente corto; dorso ligeramente curvado o convexo; grupa caída y vientre abultado; cola de inserción baja; patas largas y cerdas abundantes, largas y fuertes, su piel y cerdas deben ser de color negro. A su vez los caracteres de exclusión, según Buxade y Daza (2001), afirman que el prototipo racial de la raza ibérica no admite animales con manchas blancas en la piel, capa de piel entre blanco y negro o pintas rojizas prognatismo acusado, orejas erguidas, hernias, criptorquidia o monorquidia e infantilismo genital.

\section{RESULTADOS}

Para las variables morfométricas; longitud de la cabeza (LK), los cerdos criollos presentaron una media de 24,62 cm, identificando semovientes con rasgos que van desde 19 a $26 \mathrm{~cm}$, con una desviación estándar de 1,76 Tabla III. Al realizar el análisis de frecuencias se estimó que el 26,38\% corresponde a 19 cerdos del total de la muestra, los cuales registraron mayor longitud en la cabeza, con un promedio de 24,51 cm Figura 1. 
Tabla I. Variables zoométricas propuestas por Aparicio, (1965) y Sotillo y Serrano (1985) (Díaz Montilla, 1965) (Vieites, 1986) y recomendado por (Hurtado et al., 2004) y procedimiento de medición para obtenerlas de los animales. (Zoometric variables proposed by Aparicio, (1965) y Sotillo y Serrano (1985) (Díaz Montilla, 1965) (Vieites, 1986) y recomendado por (Hurtado et al., 2004) and measuring procedure to obtain them from the animals).

VARIABLE ZOOMÉTRICAS
Longitud de la cabeza (LK)
Longitud de la cara (LC)
Ancho de la cabeza (AK)
Ancho de la oreja (AO)
Longitud de la oreja(LO)

Alzada a la cruz (AC)

Alzada a la grupa $(A G)$

Diámetro longitudinal (DL)

Diámetro dorso esternal (DDE)

Diámetro bicostal (DB)

Ancho de la grupa (ANG)

Longitud de la grupa (LG)

Perímetro torácico $(\mathrm{PT})$

\section{MEDIDAS}

Desde la protuberancia occipital externa hasta la punta del hocico

Desde la sutura frontonasal hasta la punta del hocico.

Entre ambas apófisis cigomáticas del temporal

Tomada desde el borde superior hasta el borde inferior, pasando por el centro de la oreja.hasta la punta del mismo.

Tomada desde la punta extrema de la oreja hasta la base de inserción con la cabeza.

Medida desde el suelo hasta el punto más culminante de la cruz.

Desde el suelo hasta la tuberosidad ilíaca externa.

Distancia existente desde la articulación escápula-humeral (región del encuentro) hasta la punta de la nalga.

Distancia existente entre el punto de mayor declive de la cruz y el punto de mayor curvatura del esternón.

Distancia entre ambos planos costales, tomando como referencia los límites de la región costal con los del miembro anterior.

Es la distancia entre ambas tuberosidades iliacas externas.

Desde la tuberosidad iliaca externa (punta del anca) hasta la punta de la nalga.

Es la medida del contorno del tórax, desde la parte más declive de la base de la cruz, pasando por la base ventral del esternón y volviendo a la base de la cruz, formando un círculo recto alrededor de los planos costales.

Longitud del círculo recto que se forma en el tercio superior de la caña, rodeando el tercio del metacarpiano

Perímetro de la caña $(P C)$

Tabla II.Variables índices zoométricos propuestos por Aparicio, (1965) y Sotillo y Serrano (1985) (Díaz Montilla, 1965) (Vieites, 1986) y recomendado por (Hurtado et al., 2004) y procedimiento de medición para obtenerlas de los animales. (Zoometric index variables proposed by Aparicio, (1965) y Sotillo y Serrano (1985) (Díaz Montilla, 1965) (Vieites, 1986) y recomendado por (Hurtado et al., 2004) and measuring procedure to obtain them from the animals).

\section{VARIABLES ÍNDICES ZOOMETRICOS}

Índice cefálico

Índice de proporcionalidad

Índice corporal

Índice pelviano

Índice torácico

Profundidad relativa del pecho

Índice en metacarpo torácico

\section{MEDIDAS}

Expresado en \% como el cociente entre el ancho de la cabeza por 100 y la longitud de la cabeza.

Expresado en \% como el cociente entre la alzada a la cruz por 100 y el diámetro longitudinal.

Expresado en \% como el cociente entre el diámetro longitudinal por 100 y el perímetro torácico.

Expresado en \% como el cociente entre el ancho de la grupa por 100 y la longitud de la grupa.

Expresado en \% como el cociente existente entre el diámetro bicostal por 100 y el diámetro dorsoesternal.

Expresado en \% como el cociente existente entre el diámetro dorsoesternal por 100 y la alzada a la cruz.

Expresado en \% como el cociente existente 
La variable longitud de la cara (LC) de los cerdos criollos en estudio pertenecientes al cantón Guamote fue de $15,11 \mathrm{~cm}$, identificando semovientes con rasgos que van desde 12 a $21 \mathrm{~cm}$, con una desviación estándar de 1,49 cm Tabla III. En el análisis de frecuencias se determinó que del $100 \%$ de los porcinos criollos evaluados, el $41,66 \%$ que corresponde a 30 semovientes registraron una mayor longitud de la cara con un promedio de 14,84 cm Figura 2.

Lo que corresponde a ancho de la cabeza (AK), se obtuvo un promedio de $14,77 \mathrm{~cm}$ identificando cerdos criollos con rasgos que van desde $11 \mathrm{a} 20 \mathrm{~cm}$, con una desviación estándar de 1,57 cm Tabla III. Para el análisis de frecuencia se estimó que el 26,38 \% que corresponde a 19 cerdos criollos de un total de 72 individuos que representaron el tamaño de la muestra registran un mayor ancho en de cabeza con un valor de 14,66 cm Figura 3.
El ancho de la oreja (AO) de los cerdos criollos en el presente estudio, reportó un promedio de $15,71 \mathrm{~cm}$, identificando porcinos con rasgos que van desde 13 a $20 \mathrm{~cm}$, con una desviación estándar de 2,33 cm Tabla III. Con un análisis de frecuencia en el que 26,38 \% correspondiente a 19 cerdos criollos de un total de 72 individuos que representaron el tamaño de la muestra registran un mayor ancho de la oreja con un valor de $19,20 \mathrm{~cm}$, Figura (4-3).

La longitud de la oreja (LO), en los cerdos criollos presentaron una media de $19,56 \mathrm{~cm}$, con promedios que van desde 15 a $27 \mathrm{~cm}$, con una desviación estándar de 2,33 cm Tabla III. En el análisis de frecuencias se determinó que del $100 \%$ de los porcinos criollos evaluados el 29,16 \% que corresponde a 21 semovientes registraron una mayor longitud en las orejas con 19,71 $\mathrm{cm}$.

Para la alzada a la cruz (AC) Tabla III, se observa que los cerdos criollos del cantón Guamote presentan

Tabla III. Características zoométricas del cerdo criollo del Cantón Guamote de Ecuador (Zoometric traits of the Creole pigs that are called Pillareño of the Canton Guamote from Ecuador).

\begin{tabular}{|c|c|c|c|c|}
\hline Variables Zoométricas & Media & Desv. Est. & Mínimo & Máximo \\
\hline Longitud de la cabeza(LK) & 24,64 & 1,76 & 19,00 & 29,00 \\
\hline Longitud de la cara(LC) & 15,11 & 1,49 & 12,00 & 21,00 \\
\hline Ancho de la cabeza (AK) & 14,77 & 1,57 & 11,00 & 20,00 \\
\hline Ancho de la oreja $(\mathrm{AO})$ & 15,71 & 2,33 & 13,00 & 20,00 \\
\hline Longitud de la oreja (LO) & 19,56 & 2,33 & 15,00 & 27,00 \\
\hline Alzada de la cruz (AC) & 59,54 & 4,92 & 47,70 & 77,00 \\
\hline Alzada de la grupa(AG) & 65,92 & 4,49 & 57,00 & 83,50 \\
\hline Diámetro longitudinal (DL) & 81,61 & 6,07 & 62,00 & 102,00 \\
\hline Diámetro dorso esternal (DDE) & 31,40 & 2,54 & 23,20 & 40,00 \\
\hline Diámetro bicostal (DB) & 26,94 & 3,97 & 19,00 & 36,50 \\
\hline Ancho de la grupa (ANG) & 25,02 & 4,26 & 17,30 & 35,50 \\
\hline Longitud de la grupa (LG) & 25,85 & 2,83 & 18,00 & 33,00 \\
\hline Perímetro torácico (PT) & 91,24 & 5,30 & 76,50 & 110,00 \\
\hline Perímetro de la caña $(P C)$ & 16,91 & 1,61 & 14,00 & 24,00 \\
\hline Peso(W)= Kg & 59,61 & 8,25 & 46,00 & 84,00 \\
\hline
\end{tabular}

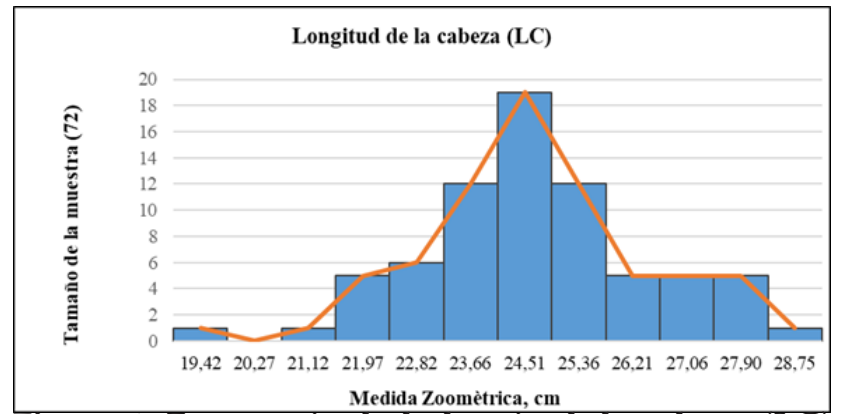

Figura 1. Frecuencia de la longitud de cabeza (LC), de cerdos criollos del cantón Guamote, Ecuador (Head length frequency of the Creole pigs that are called Pillareño of the Canton Guamote from Ecuador).

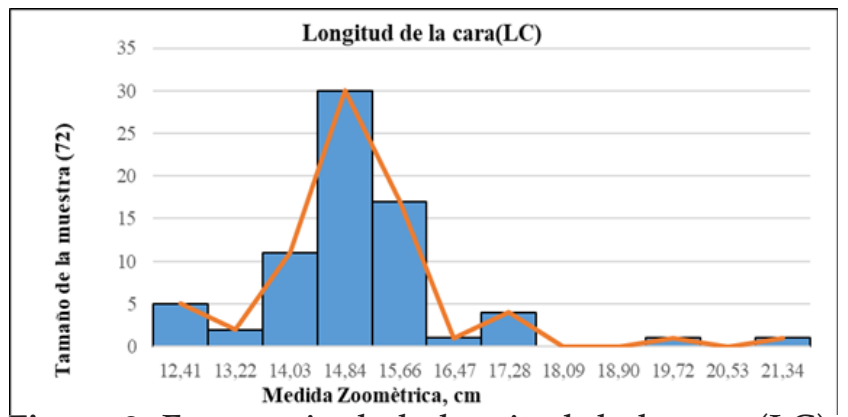

Figura 2. Frecuencia de la longitud de la cara (LC), de cerdos criollos del cantón Guamote, Ecuador (Face length frequency of the Creole pigs that are called Pillareño of the Canton Guamote from Ecuador). 
una media de $59,54 \mathrm{~cm}$, identificando cerdos con rasgos que van desde 47,70 a $77 \mathrm{~cm}$, con una desviación estándar de 4,92 cm. Al realizar el análisis de frecuencia se determinó que el 25\% correspondiente a 18 cerdos de un total de la muestra de 72 semovientes registraron mayor valor con 57,79 cm Figura 5.

En el análisis de la variable alzada de la grupa (AG), se obtuvo una media de $65,92 \mathrm{~cm}$ identificando semovientes con rasgos que van desde 57 a $83,50 \mathrm{~cm}$, con una desviación estándar de 4,49 cm Tabla III. En el análisis de frecuencias se determinó que del 100\% de los porcinos criollos evaluados el 30,55 \% que corresponde a 22 semovientes registraron una mayor alzada a la grupa correspondiente a $64,02 \mathrm{~cm}$.

Para la variable diámetro longitudinal (DL), los cerdos criollos presentaron una media de $81,61 \mathrm{~cm}$, identificando porcinos con rasgos que van desde 62 a $102 \mathrm{~cm}$, con una desviación estándar de 6,07 cm Tabla
III. Al realizar el análisis de frecuencia se determinó que el $27,77 \%$ correspondiente a 20 cerdos de un total de la muestra de 72 semovientes en estudio registraron mayor valor con $81,23 \mathrm{~cm}$.

El diámetro dorso esternal (DDE) en la presente investigación registro una media de $31,40 \mathrm{~cm}$, identificando semovientes con rasgos que van desde 23,20 a $40 \mathrm{~cm}$, con una desviación estándar de 2,54 cm Tabla III. El análisis de frecuencia determinó que el 30,55 \% correspondiente a 22 cerdos con $30,81 \mathrm{~cm}$.

Los cerdos criollos del cantón Guamote presentan una media de diámetro bicostal de $26,94 \mathrm{~cm}$, con rangos que oscilan entre 19 a 36,50 cm y desviación estándar de 3,97 cm Tabla III. En el análisis de frecuencias estimamos que el $25 \%$ correspondiente a 18 cerdos con un promedio de $27,08 \mathrm{~cm}$.

Tabla III. Características zoométricas del cerdo criollo del Cantón Guamote de Ecuador (Zoometric traits of the Creole pigs that are called Pillareño of the Canton Guamote from Ecuador).

\begin{tabular}{lllll}
\hline Variables Zoométricas & Media & Desv. Est. & Mínimo & Máximo \\
Longitud de la cabeza(LK) & 24,64 & 1,76 & 19,00 & 29,00 \\
Longitud de la cara(LC) & 15,11 & 1,49 & 12,00 & 21,00 \\
Ancho de la cabeza (AK) & 14,77 & 1,57 & 11,00 & 20,00 \\
Ancho de la oreja (AO) & 15,71 & 2,33 & 13,00 & 20,00 \\
Longitud de la oreja (LO) & 19,56 & 2,33 & 15,00 & 27,00 \\
Alzada de la cruz (AC) & 59,54 & 4,92 & 47,70 & 77,00 \\
Alzada de la grupa(AG) & 65,92 & 4,49 & 57,00 & 83,50 \\
Diámetro longitudinal (DL) & 81,61 & 6,07 & 62,00 & 102,00 \\
Diámetro dorso esternal (DDE) & 31,40 & 2,54 & 23,20 & 40,00 \\
Diámetro bicostal (DB) & 26,94 & 3,97 & 19,00 & 36,50 \\
Ancho de la grupa (ANG) & 25,02 & 4,26 & 17,30 & 35,50 \\
Longitud de la grupa (LG) & 25,85 & 2,83 & 18,00 & 33,00 \\
Perímetro torácico (PT) & 91,24 & 5,30 & 76,50 & 110,00 \\
Perímetro de la caña (PC) & 16,91 & 1,61 & 14,00 & 24,00 \\
Peso(W)= Kg & 59,61 & 8,25 & 86,00 & 84,00 \\
\hline
\end{tabular}

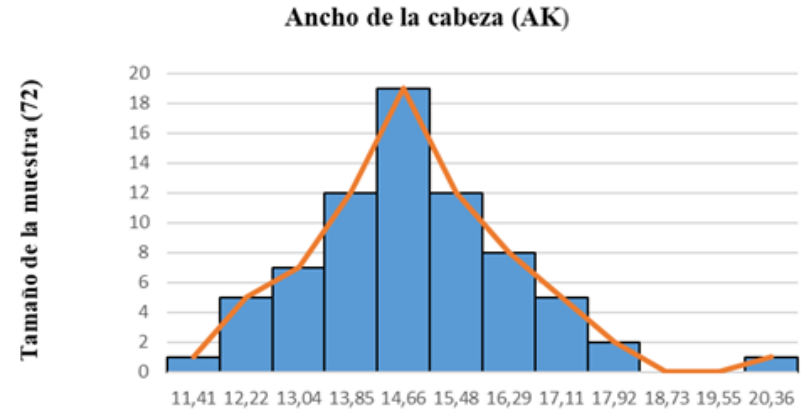

Medida Zoomètrica, cm

Figura 3. Frecuencia del ancho de la cabeza (AK), de cerdos criollos del cantón Guamote, Ecuador (Head width frequency of the Creole pigs that are called Pillareño of the Canton Guamote from Ecuador).

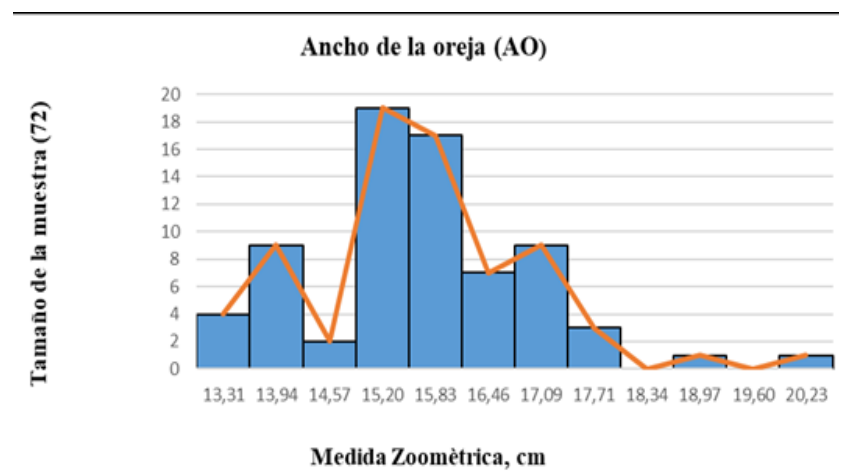

Figura 4. Frecuencia del ancho de la oreja (AO), de cerdos criollos del cantón Guamote, Ecuador (Ear width frequency of the Creole pigs that are called Pillareño of the Canton Guamote from Ecuador). 


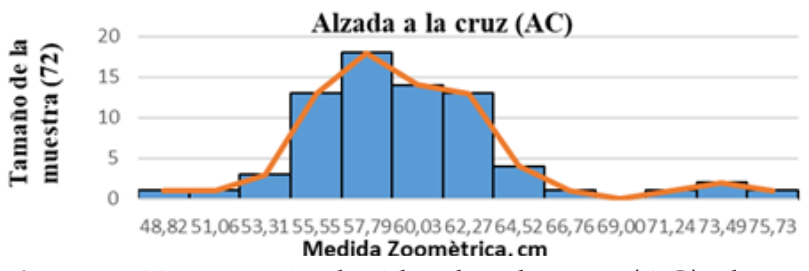

Figura 5. Frecuencia de Alzada a la cruz (AC), de cerdos criollos del cantón Guamote, Ecuador (Raised to the cross frecuency of the Creole pigs that are called Pillareño of the Canton Guamote from Ecuador).

El ancho de la grupa (ANG) en los cerdos criollos en estudio, presentaron una media de $25,02 \mathrm{~cm}$, identificando semovientes con rasgos que van desde 17,30 a $25,50 \mathrm{~cm}$, con una desviación estándar de 4,26 cm Tabla III. Para el análisis de frecuencias se determinó que del $100 \%$ de los porcinos criollos evaluados el $19,44 \%$ que corresponde a 14 semovientes registraron $25,78 \mathrm{~cm}$.

En cuanto a la longitud de grupa se registró una media de $25,85 \mathrm{~cm}$; identificando porcinos criollos con rasgos que van desde 18 a $33 \mathrm{~cm}$, con una desviación estándar de 2,83 cm Tabla III. El 41,66\% registraron valores de 26,22 y $27,47 \mathrm{~cm}$.

Para Perímetro torácico $(\mathrm{PT})$, se presentó una media de $91,24 \mathrm{~cm}$, con rasgos que van desde 76,50 a $110 \mathrm{~cm}$, con una desviación estándar de $5,30 \mathrm{~cm}$. El $25 \%$ de los animales presenta valores de $89,97 \mathrm{~cm}$.

En el perímetro de la caña en los cerdos criollos del catón Guamote, se registró una media de 16,91 cm, con rasgos que van desde 14 a $24 \mathrm{~cm}$, con una desviación estándar de 1,61 cm Tabla III. El 34,72 \% de los semovientes registraron valores de $17,81 \mathrm{~cm}$.

Para el peso vivo de los cerdos criollos, se presentaron promedios de $59,61 \mathrm{~kg}$ valores que van desde $40 \mathrm{a}$ $84 \mathrm{~kg}$, con una desviación estándar de 8,25 kg. El $25 \%$ obtuvo pesos de $56,18 \mathrm{Kg}$.

$\mathrm{Al}$ realizar el cálculo de cada índice zoométrico, se determinó que para el cefálico se obtuvo un promedio de $60,20 \%$, con valores que oscilan entre 47,82 a 87,00 $\%$, y una desviación estándar de 7,50 \% Tabla IV. A su vez el 34,72\% registraron un mayor valor en el ICF con $61,29 \%$

El índice de proporcionalidad presentó promedios de 73,18 \%, se identificó cerdos criollos con índices que van desde 64,70 a 90,60 \%, con una desviación estándar de 4,70 \% Tabla IV. El 20,83\% de los porcinos registraron un mayor IPD que corresponde a 73,44\%.

Para la variable índice corporal se observó un promedio de $89,56 \%$, que van desde 67,740 a $104,76 \%$, con una desviación estándar de 6,20\% Tabla IV. El 33,33\% del total de los animales registraron mayor valor en el ICP $90,65 \%$.

El índice pelviano alcanzó una media de 98,31\%, con valores que van desde 61,30 a $172,22 \%$, con una desviación estándar de 22,12 \% Tabla IV. Este índice indica la relación que existe entre el ancho y longitud de pelvis, los animales en estudio registraron una pelvis más larga que ancha lo cual clasifica a los semovientes como porcinos convexilíneos al tener un valor inferior a $100 \%$, es importante indicar que a medida que el largo de la grupa se incrementa sobre el ancho, el valor del índice decrece. El 47,22\% de los porcinos en estudio registraron mayor valor en el IPV de 97,49\%.

En cuanto al índice torácico se registró un promedio de $85,73 \%$ con valores que van desde 64,26 a 125 $\%$, con una desviación estándar de 10,26 \% Tabla IV. Mediante la sistemática baroniana clasifica menor o igual al $85 \%$ en un animal brevilíneo, caracterizado por ser más corto y ancho en la relación dorso esternal y bicostal. El 32\% de la muestra registraron una mayor ITO con $88,84 \%$.

La profundidad relativa del pecho registró una media de $52,93 \%$, con valores que van desde 36,19 a 63,60 $\%$ con una desviación estándar de $4,50 \%$ Tabla IV, se puede considerar como un valor adecuado, ya que los animales en estudio presentaron un tronco profundo, lo que ayuda al alojamiento de los órganos principales, el cual se encuentra directamente relacionado con la mayor rusticidad que presentan los cerdos criollos del cantón Guamote. El 40,27\% de los semovientes en estudio registraron mayor valor en la PRP con 52,10\%.

Los valores que se registraron para índice Metacarpo Torácico fueron de $18,53 \%$ con datos que van desde 15,93 a $22,68 \%$, con una desviación estándar de $1,30 \%$, este índice relaciona el perímetro de los metacarpos con la compacidad del cuerpo, a su vez mediante el presente índice se puede conocer el grado de finura del esqueleto que es fundamental para determinar el sostén de las estructuras corporales, clasificándolo de esta manera como un animal eumétrico de tamaño mediano lo que favorece a la mantención del peso corporal en los cerdos criollos estudiados. El $43 \%$ a los animales que presentaron un mayor porcentaje en el IMT con $18,60 \%$.

$\mathrm{Al}$ analizar las variables fanerópticas, los cerdos criollos pertenecientes al cantón Guamote registraron en su totalidad un color de capa negra, es decir el 100\% de la población en estudio presentó esta característica. Así también para el color de las mucosas se registró que el $100 \%$ de los animales en estudio presentaron una coloración negra, característica de los animales criollos puros que se han adaptado a condiciones climáticas adversas, pero también la coloración de la mucosa se atribuye a caracteres hereditarios que se adquieren de padres a hijos.

En lo que corresponde a la cobertura de pelo en el cuerpo el $100 \%$ de los semovientes en estudio, presentaron pelo abundante distribuido de forma armónica en el cuerpo, no se registró presencia de mamellas; y también un perfil subcóncavo, ya que los cerdos poseen longitudes de la cara largas con una pequeña declinación. A su vez los cerdos estudiados, presentaron ausencia de sindáctilia que es una característica que se produce por fusión de la $2^{\mathrm{a}}$ y $3^{\mathrm{a}}$ falange de los dedos para crear una pezuña fusionada.

\section{DISCUSIÓN}

El promedio longitud de la cabeza (LK) es similar al registrado por (Marín, 2016), quien obtuvo una media de $23,51 \mathrm{~cm}$ en la longitud de cabeza al caracterizar 
fenotípicamente el cerdo criollo en los cantones Célica, Macara y Pindal de la provincia de Loja, pero son inferiores a la media estimada por (Falconí \& Paredes, 2011), quienes al realizar el levantamiento poblacional, caracterización fenotípica y de los sistemas de producción de los cerdos criollos en los cantones de Mejía (Pichincha) y Colta (Chimborazo) obtuvieron un promedio de $27,89 \mathrm{~cm}$ en la característica zoométrica longitud de cabeza en los cerdos pertenecientes al cantón Colta, atribuyendo estas diferencias a la genética de los cerdos criollos de cada cantón de la provincia de Chimborazo.

Los valores registrados para la variable longitud de la cara (LC), son similares a los reportados por (Yépez Llangari, 2013), quien al caracterizar porcinos criollos mestizos en la comunidad de Pungupala asistida por el proyecto Cesa Micuni, registro una media de 15,09 $\mathrm{cm}$, esta similitud se debe a las características propias de los cerdos criollos pertenecientes a la provincia de Chimborazo.

Los resultados obtenidos para la variable ancho de la cabeza, son similares a los registrados por (Marín, 2016), quien obtuvo un promedio de 13,98cm; siendo inferiores a los reportados por (Falconí \& Paredes, 2011), quienes registran una media de $17,38 \mathrm{~cm}$, estas diferencias se deben a la genética autóctona establecida para la especie porcina en el transcurso del tiempo en cada cantón de la provincia determinando cerdos criollos con la estructura cefálica más ancha para el cantón Colta.

En cuanto al ancho de oreja, se registran valores similares a los obtenidos por (Falconí \& Paredes, 2011), quienes determinaron para esta variable promedios de $15,62 \pm 2,75 \mathrm{~cm}(\mathrm{CV}=17,6 \%)$ en los cerdos criollos del cantón Mejía, siendo a la vez superiores a los promedios obtenidos en los cerdos del cantón Colta, los cuales reportaron $11,27 \pm 3,59 \mathrm{~cm}(\mathrm{CV}=31,8 \%)$.

Marìn, (2016) obtuvo un promedio de 13,56 \pm 2,04 $\mathrm{cm}$ en la variable denominada anchura de oreja, valor que es inferior al registrado en el presente estudio, estas diferencias o similitudes en la característica zoométrica en los cerdos criollos se debe a características genéticas propias de los porcinos autóctonos del Ecuador. Esta característica corrobora lo mencionado por (Hurtado, 2006), quienes manifiestan que los cerdos criollos en Latinoamérica generalmente poseen orejas anchas que además son semi erectas dirigidas oblicuamente hacia delante.

El promedio registrado para longitud de la oreja (LO), en el cantón Guamote es similar al reportado por (Falconí \& Paredes, 2011) con medias de 19,25 $\pm 3,84 \mathrm{~cm}$ ( $C V=19,93 \%)$ para el cantón Mejía en Pichincha, e inferior al promedio obtenido en el cantón Colta (Chimborazo) el mismo que fue de $15,83 \pm 4,95 \mathrm{~cm}(\mathrm{CV}=31,3 \%)$. (Marín, 2016) registra un promedio de 17,42 $\pm 2,99 \mathrm{~cm}$, para la característica zoométrica longitud de oreja el cual es inferior en comparación con el presente estudio. Y que a su vez corrobora lo mencionado por Hurtado, Gonzàles y Lyca, (2004), quienes manifiestan que los cerdos criollos en Latinoamérica poseen orejas largas, además (Escobar, 2007) manifestó que los cerdos criollos en el Ecuador poseen orejas de grandes longitudes, característica que está directamente relacionada con su origen el cerdo Ibérico.

El promedio registrado en los cerdos criollos del cantón Guamote para la variable alzada a la cruz es superior a los valores obtenido por (Yépez Llangari, 2013) registro una media de 50,19 cm para los cerdos criollos, que a diferencia de los cerdos mestizos presentaron una media de 50,50 cm. (Falconí \& Paredes, 2011), obtuvieron promedios de 53,93 $\pm 8,24 \mathrm{~cm}(\mathrm{CV}=15,29 \%)$, valor similar al presente estudio, pero se registraron valores superiores en la misma investigación en los cerdos criollos del cantón Mejía en la provincia de Pichincha con valores de $65,29 \pm 10,92 \mathrm{~cm}$. Al observar el Figura 5, los valores obtenidos en el presente estudio para esta variable concuerdan con lo descrito por (Benítez \& Sánchez, 2001) quien, al realizar estudios en los cerdos criollos ecuatorianos, argumento que estos han conservado sus características debido que el campesino ecuatoriano ha preferido mantener a sus animales en "estado puro", dadas las bondades zootécnicas de los mismos, registrando alturas a la cruz que van de 47 a $59 \mathrm{~cm}$.

Los promedios registrados para la variable alzada de la grupa (AG) son superiores a los obtenidos por Marìn, (2016), quien obtuvo una media de 58,22 \pm $8,42 \mathrm{~cm}$. Mientras que Falconi y Paredes, (2011), registraron promedios de 67,77 $\pm 11,92(\mathrm{CV}=17,59 \%)$ y $53,93 \pm 8,24 \mathrm{~cm}(\mathrm{CV}=15,29 \%)$. Las diferencias de los valores para esta variable se deben a la relación directa de la estatura de los porcinos con la grupa, que además favorece a la adaptación a los diferentes sistemas de producción extensivos utilizados en traspatio.

Tabla IV. Índices zoométricas del cerdo criollo del Cantón Guamote de Ecuador (Zoometric index of the Creole pigs that are called Pillareño of the Canton Guamote from Ecuador).

\begin{tabular}{lcccc}
\hline Índices Zoométricos & Media & Desv. Est. & Mínimo & Máximo \\
Índice Cefálico(ICF), \% & 60,20 & 7,50 & 47,82 & 87,00 \\
Índice de Proporcionalidad (IPD),\% & 73,10 & 4,70 & 64,70 & 90,60 \\
Índice Corporal (ICP), \% & 89,56 & 6,20 & 67,40 & 104,76 \\
Índice Pelviano (IPV), \% & 98,31 & 22,12 & 61,30 & 172,22 \\
Índice Torácico & 85,73 & 10,26 & 64,26 & 125,00 \\
Profundidad Relativa del Pecho & 52,93 & 4,50 & 36,19 & 63,60 \\
Índice en Metacarpo Torácico & 18,53 & 1,30 & 15,93 & 22,68
\end{tabular}


Los promedios de diámetro longitudinal (DL) $\mathrm{cm}$ del presente estudio son similares a los reportados por (Yépez Llangari, 2013) quien registró una media de $81,24 \mathrm{~cm}$ de diámetro longitudinal para los cerdos criollos de la comunidad, siendo a su vez superiores a los resultados obtenidos por (Marín, 2016), quien obtuvo promedios de 64,12cm. A su vez (Falconí \& Paredes, 2011) reportan medias de $95,46 \pm 14,26 \mathrm{~cm}(14,9 \%)$ para el cantón Mejia y 73,37 $\pm 10,90 \mathrm{~cm}(\mathrm{CV}=14,85 \%)$, en los cerdos criollos del cantón Colta, valores que son superiores a los registrados en la presente investigación, lo que nos permite inferir que los cerdos criollos en el cantón Guamote poseen un diámetro longitudinal menor probablemente a que dicha característica se encuentra fija.

En referencia a diámetro dorso esternal (DDE), los resultados obtenidos en la presente investigación son similares a los reportados por (Falconí \& Paredes, 2011), quienes obtuvieron medias para el cantón Colta de $30,13 \pm 9,45 \mathrm{~cm}(\mathrm{CV}=31,5)$, probablemente por la cercanía geográfica con el Cantón Guamote. Sin embargo, (Escobar, 2007) al estudiar cerdos criollos del cantón Chambo perteneciente a la misma provincia de Chimborazo, presenta valores cercanos: Ainchi 30,34cm; Rosario 32,50cm; San Francisco 33,67cm. Pudiendo indicar que los cerdos criollos en la provincia en mención poseen características zoométricas similares, en relación a la DDE; y que además esta característica determina la profundidad corporal de los porcinos que se destinarán hacia una aptitud cárnica. A diferencia de estos estudios Marìn, (2016), obtuvo promedios de $36,03 \pm 8,75 \mathrm{~cm}$, valores que son superiores a la presente investigación lo que demuestra que las características zoométricas pueden estar en relación a cada cantón y provincia del Ecuador, debido a las tres regiones agroecológicas en las cuales se divide el país con sus 5 pisos climáticos, lo que denotaría que los porcinos poseen un alto potencial de adaptación a las diferentes condiciones ambientales. (Tixier-Boichard, Bordas, \& Rognon, 2009), (Alemu \& Tadelle, 1997).

Los resultados de diámetro bicostal del presente estudio son similares a los registrados por Escobar, (2007), obtuvo promedios de en Titaycum 26,00 cm; Guallabamba 26,68 cm; Ainchi 24,92 cm; Puculpala $26,35 \mathrm{~cm}$, en semovientes de uno a tres años de edad. A diferencia de (Véliz et al., 2009), quien registra promedios 25,12 $\pm 5,14 \mathrm{~cm}$ para los animales del cantón Valencia de la provincia de los Ríos y 24,58 $\pm 5,59 \mathrm{~cm}$, en los cerdos criollos del cantón La Maná en la provincia de Cotopaxi, siendo estos valores menores al del presente estudio. Esta característica zoométrica se encuentra directamente relacionada con la capacidad pulmonar de los semovientes, por lo cual las similitudes registradas en la provincia de Chimborazo se deben a que los cerdos criollos se encuentran en condiciones ambientales similares 3000m.s.n.m, a diferencia de los valores reportados en los cantones Valencia (105 m s. n. m.) y la Mana (altura variable de 200 y 1150 m.s.n.m.) que al encontrarse a diferentes altitudes y condiciones ambientales el diámetro bicostal de los cerdos criollos en estudio es menor.

En referencia a los resultados obtenidos para anchura de grupa en la presente investigación, estos son su- periores a los reportados por (Falconí \& Paredes, 2011), quienes obtuvieron para los cantones Colta y Mejia medias de $23.02 \pm 3.2(13.92 \%)$ y $24.13 \pm 5.38(22.31 \%)$, respectivamente. Al igual que (Marín, 2016), quien registró un promedio de $17,03 \pm 4,50 \mathrm{~cm}$. Como se puede observar en el Figura 6 los resultados obtenidos por (Buxadé Carbó \& Daza Andrada, 2001) describen que la raza ibérica de los cuales provienen los cerdos criollos de América, presentan tipos de animales longilíneos con gran musculatura en piernas y muslos en relación a otras variedades, lo que nos hace suponer que el ancho de grupa tendrá un valor alto, concordando con los datos registrados en el presente estudio.

En referencia a los resultados de longitud de la grupa obtenidos en la presente investigación, son similares a los reportados por (Falconí \& Paredes, 2011), quienes obtuvieron medias de 25,56 $\pm 5,12 \mathrm{~cm}(\mathrm{CV}=20,09 \%)$ y $24.13 \pm 5.38(22.31 \%)$ en los cantones Mejia y Colta respectivamente. (Escobar, 2007), obtuvo promedios: Titaycum 20,28cm; Guallabamba 23,40cm; El Rosario 20,05cm; Llucud $14,70 \mathrm{~cm}$. Con respecto a los resultados obtenidos (Buxadé Carbó \& Daza Andrada, 2001) especifica que la raza ibérica de la cual probablemente proviene el cerdo criollo ecuatoriano, presenta una grupa inclinada lo que tiene gran influencia sobre la longitud de grupa en relación a otras estirpes o ecotipos.

Los promedios registrados en el presente estudio para la variable perímetro torácico muestran una superioridad a los valores reportados por (Marín, 2016), quien al caracterizar fenotípicamente el cerdo criollo en la provincia de Loja, registro un promedio de 77,15 \pm $13,70 \mathrm{~cm}$. Al igual que los valores adquiridos por (Falconí \& Paredes, 2011), quienes registraron promedios de PT de 76,41 $\pm 21,65 \mathrm{~cm}$ en el cantón Mejía y 79,20 \pm $13,10 \mathrm{~cm}$ para los cerdos criollos pertenecientes al cantón Colta. De acuerdo a los resultados obtenidos en el presente estudio se reafirma lo descrito por (Arredondo Botero, 2013), (Zhenia Reyes, Isabel Santana, Neylin Sánchez, Brache, \& Camino, 2019) quienes manifiestan que los cerdos criollos poseen PT grandes debido a la características que se han fijado en ellos por interacción del genotipo de acuerdo al medio ambiente en el cual se han desarrollado, además de encontrarse relacionados directamente con razas de cerdos europeos de los cuales provienen, a la vez manifiestan que esta medida morfométrica es un indicador relacionado directamente con el peso corporal de los porcinos.

Los resultados obtenidos en el presente estudio en cuanto a perímetro de la caña son similares a los registrados por (Falconí \& Paredes, 2011), quienes reportan medias de $16,67 \pm 2,02 \mathrm{~cm}(\mathrm{CV}=12,12 \%)$ para el cantón Mejía y 13,12 $\pm 3,32 \mathrm{~cm}$. ( $C V=25,3 \%)$ para el cantón Colta. Los resultados obtenidos para esta variable ratifican lo mencionado por (Escobar, 2007), quien manifiesta que, como un proceso de adaptación por efecto del peso corporal de los animales, los huesos de la caña incrementaron su grosor.

Los resultados obtenidos para peso vivo muestran superioridad al ser comparados con investigaciones realizadas por (Benítez \& Sánchez, 2001), quien reportó pesos entre 40 y $35 \mathrm{~kg}$. A diferencia de (Falconí \& Paredes, 2011), quienes reportaron pesos vivos en los cerdos 
criollos en los cantones Colta y Mejía de 42,47 $\pm 8,35$ $\mathrm{kg}$ y 80,65 $\pm 31,5 \mathrm{~kg}$, respectivamente, además (Marín, 2016), quien al caracterizar fenotípicamente el cerdo criollo en la provincia de Loja, registro un promedio de $52,44 \pm 21,71 \mathrm{~kg}$, para la característica zoométrica peso vivo. Respecto a los resultados obtenidos en el desarrollo de la investigación se puede inferir que el cerdo criollo es un animal rústico, genéticamente de baja conversión alimenticia, pero aquí se puede diferenciar claramente como el ambiente representa el $70 \%$ en la expresión de las aptitudes productivas en los cerdos criollos del cantón Guamote, además se puede visualizar como en el trascurso del tiempo esta característica se ha ido modificando e incrementando atribuyendo esta cambio a las modificaciones en cuanto al manejo de la alimentación en sistemas que van desde extensivo a semi-extensivo.

En términos zootécnicos, se designa índice a la correspondencia existente entre dos dimensiones locales y de las proporciones existentes entre las mismas, tratando de enunciar con su uso las proporciones y conformación general de los animales (diagnosis racial), así como estados corporales que inducen al animal a determinadas funcionalidades o la evaluación del grado de rendimiento que posee para una aptitud determinada (Arredondo Botero, 2013).

Los resultados obtenidos de índice cefálico en la presente investigación son similares a los registrados por (Marín, 2016) quien, al caracterizar fenotípicamente el cerdo criollo en la provincia de Loja, obtuvo un promedio de 60,26 $\pm 11,64 \%$ en el ICF. A diferencia de los cerdos criollos pertenecientes al cantón Valencia de la provincia de Los Ríos y al cantón La Mana de Cotopaxi donde se obtuvo 45,89 $\pm 6,68$ y 51,15 \pm 7,94 \% respectivamente; resultados presentados por (Estupiñán et al., 2009), al realizar el estudio morfoestructural de una población de cerdos naturalizados y que a su vez son inferiores en comparación al presente estudio. (Falconí \& Paredes, 2011), registraron en el cantón Mejía un ICF de 57,12 \pm 9,68 y en el cantón Colta de 63,45 $\pm 13,68$ valores que son superiores a los obtenidos. En concordancia a los resultados obtenidos por (Herrera \& Luque, 2009), manifestaron que los caracteres étnicos referidos por la cabeza, como el índice cefálico tienen su importancia genética y etnológica, sobretodo porque su variación no está influenciada por los factores ambientales ni de manejo.

El índice de proporcionalidad, expresa la relación que existe entre la alzada a la cruz y el diámetro longitudinal definiendo la rectangularidad del cuerpo del animal, a medida que el valor se aleja del $100 \%$, los animales serán más rectangulares, algunos autores consideran que es mejor si excede de $50 \%$, en este estudio los cerdos criollos presentaron formas rectangulares, forma predominante en los animales de aptitud carnicera. Respecto a los resultados obtenidos (Escobar, 2007), registró en los cerdos criollos pertenecientes al cantón Chambo promedios según las diferentes comunidades de Puculpala de 70,59; Ulpan 71,67; y El Vergel de 66,75, de la misma manera (Falconí \& Paredes, 2011), quienes al caracterizar los cerdos criollos de los cantones Mejía y Colta registraron medias para IPD de $68,71 \pm 10,57(\mathrm{CV}=15,38 \%)$ y $74,29 \pm 16,19(\mathrm{CV}=21,28 \%)$ respectivamente, valores que son similares a los del presente estudio, pero (Marín, 2016), obtuvo un promedio de 86,76 $\pm 20,84 \%$ al caracterizar fenotípicamente el cerdo criollo en la provincia de Loja, valores que son superiores a los de esta investigación. Las diferencias o similitudes entre investigaciones están relacionadas directamente con las localidades y diferentes pisos climáticas en las que se encuentran los cerdos criollos.

Los resultados obtenidos en cuanto al índice corporal son similares a los registrados por (Escobar, 2007), con $88,90 \%$ de ICP, a diferencia de los ICP registrados por (Marín, 2016) y (Estupiñán et al., 2009), quienes obtuvieron promedios de $84,32 \pm 16,39$ y $83,33 \%$ respectivamente, siendo estos valores inferiores a los del presente estudio, demostrando de esta manera la variabilidad genética del cerdo criollo en el Ecuador, según el sitio geofigura en los cuales se encuentran.

El índice pelviano indica la relación que existe entre el ancho y longitud de pelvis, los animales en estudio registraron una pelvis más larga que ancha, lo cual clasifica a los semovientes como porcinos convexilíneos al tener un valor inferior a $100 \%$, es importante indicar que a medida que el largo de la grupa se incrementa sobre el ancho, el valor del índice decrece. En relación a los resultados obtenidos de índice pelviano (Falconí \& Paredes, 2011), registraron en el cantón Mejía un IPV de 98,13 $\pm 28,87 \%$, mientras que para el cantón Colta $100,01 \pm 13,30$ valores que son superiores a los obtenidos. A diferencia de (Marín, 2016), quien registra un promedio de $87,64 \pm 41,78 \%$ en los cerdos criollos pertenecientes a los cantones Celica, Macará y Pindal de la provincia de Loja, de la misma forma (Estupiñán et al., 2009) reportaron cifras de 76,67 \pm 6,85 y 79,69 \pm 8,15 en los cerdos criollos de los cantones Valencia y La Maná respectivamente.

Los valores obtenidos de índice torácico en la presente investigación muestran superioridad en comparación con los siguientes autores (Marín, 2016), quien al caracterizar los cerdos criollos en la provincia de Loja obtuvo una media de 62,06 \pm 17,16 \%, (Falconí \& Paredes, 2011) quienes reportaron promedios de $79,20 \pm 13,10 \%$ para los cerdos criollos del cantón Colta y para Mejía 76,41 $\pm 21,65(\mathrm{CV}=28,33 \%)$.

Los valores registrados de profundidad relativa del pecho muestran superioridad a los obtenidos por (Falconí \& Paredes, 2011), al caracterizar a los cerdos criollos de los cantones Colta y Mejia con 55,24 $\pm 14,28$ y $57,48 \pm 6,50 \%$ respectivamente, siendo similares a los resultados registrados en la investigación de (Escobar, 2007) quien en las comunidades de Francisco, Guallabamba y Rosario obtuvo 56,60; 55,27 y 56,98.

Los resultados obtenidos de índice Metacarpo Torácico en la presente investigación son similares a los registrados por (Marín, 2016), quien al caracterizar los cerdos criollos en la provincia de Loja obtuvo una media de 18,48 $\pm 3,95 \%$ en el IMT; al igual que los valores de (Falconí \& Paredes, 2011), quienes en los can-

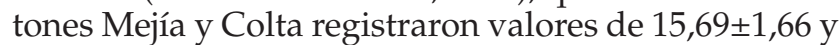
$17,14 \pm 5,27 \%$ respectivamente.

Con respecto a los resultados obtenidos (Benítez \& Sánchez, 2001) quien al realizar estudios en los cerdos 
criollos ecuatorianos, argumento que el color de la capa predominante en los cerdos criollos ecuatorianos es negro debido a la relación que posee con el cerdo ibérico de variedad Gabana; resultados similares a los reportados por (Yépez Llangari, 2013), en porcinos criollos mestizos en la comunidad de Pungupalà del cantón Alausí (Chimborazo); de la misma forma (Escobar, 2007) en el cantón Chambo, determinó una dominancia de la coloración negra en un 96,4\%, y apenas 3,6 \% presentan una capa combinada de color blanco y negro, lo que atribuyó a un mestizaje con cerdos de la raza Hampshire. Pero existen diferencia con los resultados reportados por (Marín, 2016), quien al caracterizar los cerdos criollos en la provincia de Loja obtuvo coloración negra entera en un 59\%, copa colorada un $14 \%$, un $9 \%$ de coloración negra en combinación con una franja blanca, colorada manchado $8 \%$, negro manchas blancas un 7\%, muy escasa la coloración gris con $2 \%$ y $1 \%$ colorada franca blanca. Las similitudes o diferencias entre los estudios se deben claramente a la variabilidad genética que existe entre los cerdos criollos de cada provincia del Ecuador.

En relación a los resultados para coloración de la mucosa (Marín, 2016), al caracterizar los cerdos criollos en la provincia de Loja registró el 91\% mucosa negra. A diferencia de (Falconí \& Paredes, 2011), quienes registraron en los cantones Mejía y Colta cerdos criollos con mucosas de tonalidad oscura en un $71,4 \%$. Pero existe una variabilidad en los resultados de (Estupiñán et al., 2009), quienes reportaron que en el cantón La Maná existe una gran incidencia del color de mucosa clara en $37,50 \%$, seguida de la oscura $36,36 \%$ y finalmente manchada 25,00 \%, a diferencia del cantón Valencia en donde prevalece la mucosa oscura con $40,98 \%$ seguida de la tinción clara con 27,87 \%, la manchada 18,03 \% y finalmente $13,12 \%$ la despigmentada.

En cuanto a la presencia de pelo, los resultados obtenidos son similares a los registrados por (Falconí \& Paredes, 2011), quienes registraron en la mayoría de cerdos criollos estudiados pelo abundante $92,5 \%$ y el restante 7,5 \% de pelo escaso a diferencia de (Marín, 2016), quien en los cantones de la provincia de Loja en los que se realizó su investigación (Célica, Macará y Pindal) se obtuvieron los siguientes resultados: presencia de pelo abundante $43 \%$, escaso $38 \%$ y ausencia de pelo $19 \%$; esto probablemente se deba a la interacción que ejerce la temperatura ambiental sobre el fenotipo del animal. OJO.

En cuanto a la presencia de mamellas, además estos resultados son similares a los registrados por (Falconí \& Paredes, 2011), quienes en los cantones Colta y Mejía registraron un $100 \%$ de ausencia de mamellas, esta es una característica propia de los cerdos ibéricos. A diferencia de Marín, (2016), quien en el estudio de los cerdos criollos de los cantones Célica, Macará y Pindal, la presencia de mamellas fue del 1\% y el 99\% ausencia de las mismas.

En relación a los resultados obtenidos (Falconí \& Paredes, 2011), manifiestan que en el cantón Colta existe gran presencia de perfiles cóncavos en relación a las localidades comparadas, a su vez $1 \%$ de animales con el perfil recto. Para perfil concávo apenas un 3,8 \% se presenta en el cantón Mejía. A diferencia de (Marín, 2016), quien determinó en los cerdos criollos perfil cefálico o frontonasal en los cantones de estudio (Celica, Macará y Pindal) una presencia del 77 \% de rectilíneo, seguido del $23 \%$ de subcóncavo, y ausencia de perfil cóncavo.

En relación a los resultados obtenidos de manera única (Revidatti, 2009), registraron un cierto número de cerdos del nordeste argentino con pezuñas enteras (no hendidas), al igual que (Spilsbury, Lemus, Ramírez, \& Alonso, 2003), (Alonso, Spilsbury, Ramírez, \& Lemus, 2003) en cerdos nativos mexicanos, que a su vez ratifican que los cerdos que poseen esta característica, muestran una resistencia a la peste porcina y padecen menos problemas locomotores.

\section{CONCLUSION}

Los cerdos criollos del cantón Guamote se clasifican como animales dolicocéfalos, longilíneos o dolicomorfos, de aptitud cárnica, su estructura reproductiva presenta una grupa de líneas convexas donde predomina la longitud sobre la anchura; son porcinos brevilíneos, de buena profundidad en el pecho lo cual determina su funcionalidad y rusticidad; su relación metacarpo torácico los denomina como animales eumétricos, es decir porcinos de tamaño mediano en relación a su masa con los miembros de soporte.

Según los resultados de la investigación, el estándar del cerdo criollo del Cantón Guamote provincia de Chimborazo sería, color de capa y mucosa negra, pelo abundante distribuido de forma armónica sobre su cuerpo, ausencia de mamellas, perfil subcóncavo con una ligera depresión de la línea frontonasal en la unión de la cara con el cráneo, ausencia de sindáctilia.

Además, los cerdos criollos del Cantón Guamote, presentaron similitud morfológica con las características del cerdo ibérico.

\section{AGRADECIMIENTOS}

Los autores agradecen al proyecto "Caracterización morfológica y genética del cerdo criollo de la Provincia de Chimborazo"; a la Escuela Superior Politécnica de Chimborazo, Facultad de Ciencias Pecuarias y a la Red Iberoamericana de Conservación de la Biodiversidad de Animales Domésticos (CONBIAND).

\section{BIBLIOGRAFÍA}

Alemu, Y., \& Tadelle, D. (1997). The status of poultry research and development in Ethiopia, research bulletin No. 4, poultry commodity research program Debrezeit Agricultural research center. Alemaya University of agriculture, Ethiopia, 62.

Alonso, M. R. E. A., Spilsbury, M. A., Ramírez, N. R., \& Lemus, F. C. (2003). Características morfológicas en cerdos nativos mexicanos. Archivos de Zootecnia, 52(197), 105-108.

Aparicio, G. (1960). Exterior de los grandes animales domésticos. Imprenta Moderna. Córdoba, 5-27.

Arredondo Botero, J. V. (2013). Caracterización de los sistemas de producción tradicional, morfología y diversidad genética del cerdo criollo de la Región Pacífica colombiana. Doctorado en Ciencias Agrarias. 
Benítez, W. (1995). De la domesticación a los actuales sistemas, de producción. . Docencia, Ciencia y Cultura . 2, 159.

Benítez, W., \& Sánchez, M. D. (2001). Los cerdos locales en los sistemas tradicionales de

producción. estudio de producción y sanidad animal. FAO.(148), 208.

Buxadé Carbó, C., \& Daza Andrada, A. (2001). Porcino Ibérico: aspectos claves.

C.M. Vietes, L. R. B. (1986). Cerdos para carne (E. H. Sur Ed. Primera edición ed.).

Ca, S. Paiva SR, Pereira RW, Guimarães SE, Dutra WM, Murata LS andMariante AS 2009."Iberian origin of Brazilian local pig breeds based on Cytochrome b (MT-CYB) sequence". Anim. Genet, 40(5), 759-762.

Díaz, R. (1965). Ganado Porcino (E. Salvat Ed.). Barcelona, España.

Escobar, J. (2007). Caracterización y sistemas de producción de los cerdos criollos del Cantón Chambo. Escuela Superior Politécnica de Chimborazo.

Estupiñán, V., Vasco, D., Barreto, S., \& Zambrano, K. (2009, 2007). Estudio morfoestructural de una población de cerdos naturalizados en el cantón La Maná, provincia de Cotopáxi, Ecuador.

Falconí, C., \& Paredes, M. (2011). Levantamiento Poblacional, Caracterización Fenotípica y de los sistemas de producción de los cerdos Criollos en los Cantones de Mejía (Pichincha) y Colta (Chimborazo). Agropecuario Sangolquí. Escuela Politécnica del Ejército. Recuperado de http:www.monografias.com/trabajospdf2/manual-produccionporcicola/manual-produccion-porcicola. pdf.

Gutiérrez, R. A. S., Luna, R. G., \& Nájera, M. d. J. F. (2018). Caracterización morfológica de un rebaño de conservación de cabras criollas en Zacatecas, México. Archivos de Zootecnia, 67(257), 73-79.

Hernández-Baca, M., Gámez-Rivas, A., \& Zeledón-Ortega, Y. (2017). Caracterización morfológica del cerdo criollo (Sus scrofa domesticus) en el municipio de Nueva Guinea, RACCS. La Calera, 17(28), 21-27.

Herrera, M., \& Luque, M. (2009). Morfoestructura y sistemas para el futuro en la valoración morfológica. Valoración morfológica de los animales domésticos. Madrid, Minist. de Medio Ambiente y Medio Rural y Marino.

Hurtado, E. (2004). Evaluación preliminar del cerdo Criollo y los sistemas de producción en los estados Apure y Guárico de Venezuela.

Hurtado, E. (2006). El cerdo criollo en Venezuela. Status actual y perspectivas. línea]. Disponible en: http:www.sian.info.ve/porcinos/ publicaciones/rccpn/rev1306/hurtado. htm (Consultado: martes 28 de julio de 2009).

Marín, M. (2016). Caracterización Fenotípica del Cerdo Criollo en los Cantones Célica, Macará y Pindal de la Provincia de Loja. (Grado), Universidad Nacional de Loja.

Panés, A. (1916). Ganadería Murciana. Artes Gráficas Mateu. Murcia (España), 20-22.

Pineda, E. P. (2006). Caracterizacion genetica del cerdo criollo cubano utilizando marcadores moleculares. Revista Computadorizada de Producción Porcina Volumen, 13(suplemento 2).

Ramirez, O., Ojeda, A., Tomas, A., Gallardo, D., Huang, L. S., Folch, J. M.,... Hanotte, O. (2009). Integrating Y-chromosome, mitochondrial, and autosomal data to analyze the origin of pig breeds. Molecular biology and evolution, 26(9), 2061-2072.

Revidatti, M. A. (2009). Caracterización de cerdos criollos del Nordeste Argentino[tesis doctoral]. Córdoba, Andalucía, España: Universidad de Córdoba.

Rodero, A., Delgado, J. V., \& Rodero, E. (1992). El ganado andaluz primitivo y sus implicaciones en el descubrimiento de América. Arch Zoot, 41(154), 383-400.

Sotillo, J. L., \& Serrano, V. (1985). Producción animal I. Etnología zootécnica, 1, 111-116.

Spilsbury, M. A., Lemus, F. C., Ramírez, N. R., \& Alonso, M. R. E. A. (2003). Rendimiento reproductivo en cerdos nativos mexicanos. Archivos de Zootecnia, 52(197), 109-112.

Thornton, P. K., van de Steeg, J., Notenbaert, A., \& Herrero, M. (2009). The impacts of climate change on livestock and livestock systems in developing countries: A review of what we know and what we need to know. Agricultural systems, 101(3), 113-127.

Tixier-Boichard, M., Bordas, A., \& Rognon, X. (2009). Characterisation and monitoring of poultry genetic resources. World's Poultry Science Journal, 65(2), 272-285. doi: 10.1017/s0043933909000233

Vargas, J. C., Velázquez, F. J., \& Chacón, E. (2015). Estructura y relaciones genéticas del cerdo criollo de Ecuador. REDVET. Revista Electrónica de Veterinaria, 16(7), 1-11.

Véliz, K. E., Mora, D. V., Barreto, S., \& Zambrano, K. (2009). Estudio morfoestructural de una población de cerdos naturalizados en los Cantones Valencia y La Maná, Ecuador. Revista Ciencia y Tecnología, 2(2), 15-20

Yépez Llangari, R. E. (2013). Caracterización de los Porcinos Criollos y Mestizos en la Comunidad de Pungalá Asistida por el Proyecto CESA-MICUNI.

Zenteno, E. B. S., Cueva, L. R. S., \& Crespo, G. E. L. (2019). Calidad de la canal de cerdos en la industria porcina de ecuador (Artículo de Revisión). Revista Ecuatoriana de Ciencia Animal, 2(2), 118-131. Zhenia Reyes, Isabel Santana, Neylin Sánchez, Brache, F., \& Camino, Y. (2019). Correlaciones entre el peso vivo y dos medidas morfométricas en cerdos criollo cubano en la etapa de cría. Revista Computadorizada de Producción Porcina, 26 (2). 